\title{
Shale Porosity Determination Methods and Their Influencing Factors
}

\author{
Jilin Li \\ School of Geoscience and technology, Southwest Petroleum University, Chengdu, 610500, China
}

Keywords: Shale porosity, Nitrogen adsorption method, Mercury intrusion method, Acoustic logging; Nuclear magnetic resonance.

\begin{abstract}
The pore structure of shale gas reservoir is of important research significance to the development and exploitation of shale gas and domestic scholars have done massive researches on the pore structure of shale. To better study the porosity features of shale gas reservoir, the common porosity determination methods and their influencing factors are analyzed in this paper and the following conclusions are made: the domestic common porosity determination methods are nitrogen adsorption method, mercury intrusion method, acoustic logging method and nuclear magnetic resonance method and all of them have the factors that may affect the porosity test results. Adsorbent is of important significance to the accuracy of nitrogen adsorption method; material hardness, sample preparation and contact angle pose great influences on mercury intrusion method; the interval transit time is a decisive influencing factor in the acoustic wave test; the instrument parameter, micro pore of sample and paramagnetic substance are the main factors that affect the experimental results of nuclear magnetic resonance. I hope that this research can provide certain reference to the domestic and foreign peers.
\end{abstract}

\section{Introduction}

Porosity is an important parameter of shale gas reserves and a key indicator about whether the shale gas reservoir is effective and determines the shale gas storage capacity. The shale gas storage can be evaluated by porosity determination method. Therefore, the porosity is always the difficult and key point of shale gas reservoir research and of massive experimental research at home and abroad. There are many porosity determination methods and the methods mainly used at home are nitrogen adsorption method, mercury intrusion method, acoustic logging method and nuclear magnetic resonance method. These methods are discussed in detail and the influencing factors of porosity determination area analyzed in this paper.

\section{Porosity determination methods}

\subsection{Nitrogen adsorption method}

Nitrogen adsorption method is to conduct degassing treatment of dried shale by setting the different test pressure with experimental device and then put it in the liquid nitrogen. The shale porosity can be determined by determining the content of liquid nitrogen and finally the adsorption and desorption curve can be drawn. The size and shape of pore of shale can be determined according to the form of hysteresis loop in the curve and then the pore radius and pore diameter distribution can be calculated. The method is as follows:

(1) Calculation of pore radius. Kelvin principle is generally used for calculation in the nitrogen adsorption method and the conversion is conducted by recurrence relation expression. The equation is as follows:

$$
r_{k}=\frac{-2 \gamma V_{m}}{R T \ln \left(p / p_{0}\right)}
$$


Where: $\gamma_{\text {is }}$ the surface tension when the nitrogen gas reaches the boiling point; $V_{m}$ is the molar volume when the liquid nitrogen gas reaches the boiling point; $\mathrm{R}$ is the atmospheric gas constant; $\mathrm{T}$ is the boiling point of nitrogen gas $(77 \mathrm{~K}) ; p / p_{0}$ is the relative pressure of nitrogen gas; $r_{k}$ is the Kelvin radius of shale pore.

(2) Calculation of pore diameter distribution. Pore diameter distribution is an important parameter of porosity calculation and there are many researches with regard to it at home. At present, Barrett-Joyner-Halenda principle is generally used for the calculation of pore diameter distribution at home and abroad. The calculation equation is as follows:

$$
V_{p n}=\left[\frac{r_{p n}}{r_{k n}+\Delta t_{n} / 2}\right]^{2}\left[\Delta V_{n}-\Delta t_{n} \sum_{j-1}^{n-1} A_{c j}\right]
$$

Where: $V_{p n}$ is the porosity volume; ${ }^{r n}$ is the maximum pore radius; ${ }^{k n}$ is the capillary radius; $V_{n}$ is the capillary volume; ${ }^{t_{n}}$ is the thickness of absorbed nitrogen gas layer of the sample; ${ }^{A_{c j}}$ is the previously empty volume of sample.

For the layered micro pore model of shale, Dubinin-Astakhov principle is used for calculation. The equation is as follows:

$$
W=W_{0} \exp \left[-\left[\frac{-R T \ln p / p_{0}}{E}\right]^{n}\right]
$$

Where: $\mathrm{W}$ is the mass of absorbed nitrogen gas of the sample under pressure $p / p_{0}$ and temperature T; ${ }^{W_{0}}$ is the total mass of absorption;

$T$ is the test temperature; $E$ is the characteristic energy; $n$ is the non-integer (1 3)。 $n$ and $E$ can be obtained by the isothermal absorption curve and the pore diameter distribution can be calculated by substituting $\mathrm{n}$ and $\mathrm{E}$ into equation (2.4).

$$
\frac{d\left(\omega / \omega_{0}\right)}{d r}=3 n\left[\frac{K}{E}\right]^{n} r^{-(3 n+1)} \exp \left[-\left[\frac{K}{E}\right]^{n} r^{-3 n}\right]
$$

Where: $\mathrm{r}$ is the pore diameter; $\mathrm{K}$ is the interaction constant, $2.96 \mathrm{~kJ} \cdot \mathrm{nm} 3 / \mathrm{mol}$ 。

\subsection{Mercury intrusion method}

Mercury intrusion method is a common determination method of rock porosity because mercury is wet for most rock-forming minerals and can overcome the capillary resistance and then enter the inside of pores when it is forced to enter the rock porosity by external pressure. Finally, the pore size and distribution can be calculated according to the injection rate of mercury. At present, the conventional mercury intrusion and constant-rate mercury intrusion are generally used to determine the shale porosity at home and abroad.

The conventional mercury intrusion method means that the mercury enters the rock pores under constant pressure, in which the mercury intrusion amount is recorded to determine the rock porosity. The liquid mercury changes from a static state to another static state under the action of pressure difference. By recording the changes of mercury volume, the capillary pressure curve of rock can be drawn and then the characteristics parameters of pore structure of rock can be calculated.

The constant-rate mercury intrusion method is a new measuring method of rock porosity. It forces the liquid mercury flows into the rock at an extremely low rate by mercury injection apparatus and then determines the capillary pressure curve of rock. Due to the low rate of liquid mercury, the whole process can be regarded as a quasistatic process. The pore where the mercury flow through will have meniscus change, thus leading to the change the capillary pressure. After entering the rock pore, the features of pore in the rock can be determined according the changes of mercury. Therefore, the 
measurement difficulty and complexity are larger than the conventional mercury intrusion method, with higher accuracy. US ASPE-730 Mercury Injection Apparatus is mainly used to measure the rock porosity at home.

\subsection{Acoustic logging}

Acoustic logging method is always an important measuring method of reservoir porosity and has an important position in the geological domain. Acoustic logging mainly includes acoustic velocity logging and amplitude logging, in which the former one is to measure the propagation velocity of acoustic wave in the rock and the latter one is to study the formation cementation according to the changes of acoustic wave propagation amplitude in the rock formation. Acoustic logging is not only widely used in the shale porosity determination, but also in the porosity determination of conventional sandstone reservoir.

The practice research proves that the porosity and interval transit time have the following approximation relation in the clean formation with good consolidation:

It can be obtained:

$$
\Delta t=\Phi \Delta t_{f}+(1-\Phi) \Delta t_{m a}
$$

Equation (2.5) is called time average equation. Where: $\Delta t$ — transit time in the rock formation; $\Delta t_{f} \ldots$ _transit time of liquid in the rock pore; $\Delta t_{m a}$ _ interval transit time of rock matrix.

For specific region, the rock matrix component and liquid in the rock are fixed, so equation (2.5) is a linear equation and it can be obtained after simplification:

$$
\Delta t=A \Phi+B
$$

For different regions, the relation curve of interval transit time and porosity has significant difference. A and B in equation (2.6) can be determined by the different core analysis of porosity and interval transit time under different logging depth. Therefore, the different logging is of massive core data analysis, and then the rock reservoir model is established according to the rock data and the porosity and other relevant information are obtained. The more the core data is, the higher the reliability of model parameters will be.

\subsection{Nuclear magnetic resonance logging}

Nuclear magnetic resonance is a response of atomic nucleus to the magnetic field. When an alternating magnetic field is formed by applying a RF magnetic field in the vertical direction of stabilized magnetic field and the new magnetic field has the same vibration frequency with the proton, the low-energy proton will become the high-energy proton by absorbing energy, vice versa, thus leading to nuclear magnetic resonance. Therefore, the nuclear magnetic resonance energy depends on the number of hydrogen atoms, the larger the number is, the higher the energy will be. According to the above principle, the nuclear magnetic resonance signal strength of different porosity can be determined to judge the size of rock pore. CPMG is generally used at home to measure the transverse relaxation process of rock. The signal strength is:

$$
M(t)=M(0) \sum_{n=0}^{\infty} f_{n} e^{\left(-\frac{t}{T_{2 n}}\right)}
$$

Where, $M(t)$ is the strength of nuclear magnetic resonance signal; ${ }^{M(0)}$ is the initial amplitude of nuclear magnetic resonance signal; $f_{n}$ is the relative attenuation amplitude of $n$ transverse relaxation component; $T_{2 n}$ is the transverse relaxation time of relaxation component; 
In the rock pores, the transverse relaxation includes three relaxation mechanisms: surface relaxation, body relaxation and diffusion relaxation. Therefore, the transverse relaxation rate can be expressed as follows:

$$
\frac{1}{T_{2}}=\frac{1}{T_{2 B}}+\frac{1}{T_{2 s}}+\frac{1}{T_{2 D}}=\frac{1}{T_{2 B}}+\rho_{2} \frac{S}{V}+\frac{\left(\gamma G T_{E}\right)^{2} D}{12}
$$

Where, $T_{2 B}$ in term 1 on the right side is the body relaxation time, because $T_{2 B} \succ T_{2 s}$, so it is ignored during calculation; $T_{2 s}$ in term 2 is the surface relaxation time, in which $\rho_{2}$ is the transverse relaxation rate of solid-liquid surface; $\mathrm{S}$ is the superficial area of pore; $\mathrm{V}$ is the pore volume. In the porous media, the pore of different shape and size corresponds to different $T_{2 s}$, which should be concerned; $T_{2 D}$ in term 3 is diffusion relaxation time; $\gamma$ is the gyromagnetic ratio; $G$ is the magnetic field gradient constant; D is the molecular diffusion coefficient; ${ }_{E}$ is the echo spacing. It is clear that nuclear magnetic resonance signal contains pore structure, fluid type and other information. This method is affected by various factors in the actual measurement. To obtain accurate porosity by nuclear magnetic resonance, these influencing factors must be analyzed.

\section{Analysis of the influencing factors of porosity determination methods}

\subsection{Influencing factors of nitrogen adsorption method}

The main factors that affect the nitrogen adsorption method is the selection of adsorbent. The adsorbent is the basis for the nitrogen adsorption separation process, with greater specific surface area and selective absorption ability. Different absorbent has different absorption ability, mechanical strength and regeneration capacity as well as different absorption ability of micro pore and big pore, so it imposes great influences on nitrogen adsorption method.

The absorbent can be divided into many types by particle shape, particle size, chemical component and surface shape, such as micro-pore absorbent and coarse-pore absorbent, polar absorbent and non-polar absorbent, powdery absorbent and granular adsorbent. For example, as to the micro-pore absorbent, BET method may have great error because the micro-pore filling is formed when the relative pressure is less than 10-4in the tiny porous channel, so it cannot describe the absorption in the micro pore with the hypothesized multi-molecular layer adsorption. Based on this, researchers adopt more reasonable theory to describe the absorption process in the micro pore and calculate the specific surface area of micro pore.

\subsection{Influencing factors of mercury intrusion method}

\subsubsection{Material hardness}

Mercury intrusion method should be conducted under high pressure, so the rock material must have high hardness; otherwise, the rock sample is easily deformed and even broken under the high external pressure to damage the material structure. For example, Chinese scholar Xie Yijin determines the pressure of mercury intrusion method and the research shows that when the external pressure is up to $140 \mathrm{MPa}$, the rock volume decreases by $2 \%$. According to the principle of mercury intrusion method, the decrease part of rock volume will be regarded as the rock pore volume, thus leading to larger rock porosity. Therefore, the rock with low hardness should be of compression test to measure the volume deformation of rock under different pressure, which can be reduced from the pore volume. For the rock with low hardness, the pressure must be controlled within a smaller range, so as to keep the integrity of rock.

\subsubsection{Sample preparation}

The sample preparation also imposes great influences on the measurement results of mercury intrusion method. Mercury intrusion method requires that the sample should have a high vacuum, so 
the rock sample must be fully dried, without any volatile water. If the concrete contains constitution water, samples will have irreversible changes during drying, for example, if the cement concrete contains gel pore and the pore contains constitution water, the overflow of partial volatile constitution water may generate gel microcrystalline hole during drying. The cement sample should be not hydrated immediately after sampling and then dried under low temperature.

\subsubsection{Contact angle}

The contact angle imposes great influences on the measurement results of mercury intrusion method. The contact angle of mercury and rock surface, material composition and roughness of rock as well as cleanness of mercury have great influences. Domestic and foreign scientists have done in-depth research of contact angle. For example, Ritter and Drake take $140^{\circ}$ as the contact angle of mercury intrusion method. The massive experiments show that this method has a great error of more than $50 \%$ because the mercury porosity apparatus has higher requirements on the contact angle. Therefore, the contact angle of rock sample must be of qualitative or quantitative analysis to minimize the error. The scanning curve, dynamic contact angle or distribution curve can be used to measure the contact angle of rock.

\subsection{Influencing factors of acoustic logging}

In the acoustic logging, the interval transit time imposes great influences on the measurement of pore. In the acoustic wave propagation in the rock pore, the interval transit time can reflect the size and number of pores in the rock, so the factors that affect the interval transit time also affect the measurement of rock porosity. The fill material in the rock pore is mainly muddy and the liquid is mainly water, gas and oil. The transit time of acoustic wave in different substances is different.

The interval transit time of acoustic logging is the weighted average of the interval transit time of substances in the rock and imposes great influences on the test results, and that of liquid is larger than the rock, so the samples must be dried. The shale content of the sample and the variable compaction range of sample are larger. According to the relevant research, the interval transit time of uncompressed rock mud stone is up to $600 \mathrm{~ms} / \mathrm{m}$ and that of mud stone with a large buried depth is only $300 \mathrm{~ms} / \mathrm{m}$. Therefore, the interval transit time of different depth should be of different treatment so as to ensure the experimental effectiveness.

\subsection{Influencing factors of nuclear magnetic resonance logging}

\subsubsection{Instrument parameter}

Nuclear magnetic resonance technology has higher requirements and the selection of instrument parameter will determine the accuracy of final results. RecCore04 rock sample analyzer is generally adopted at home. The key parameter of the instrument is the echo spacing, which imposes great influences on the relaxation time. According to equation (2.9), the larger the influence of by diffusion term is, the larger and the smaller the surface relaxation rate will be. Therefore, the influence of diffusion term must be reduced by reducing the echo spacing.

\subsubsection{Micro pore of sample}

Micro pore has certain bound liquid, which may shorten the transverse relaxation time to reduce the rock porosity detected by nuclear magnetic resonance. As the echo spacing of instrument is short, the information of the bound water of micro pore is hard to be detected, thus leading to wrong feedback information. Therefore, the full drying imposes great influences on the final test results of sample.

\subsubsection{Paramagnetic substance}

Nuclear magnetic resonance technology obtains the information of pore by magnetic field. If the paramagnetic ions of sample are in the magnetic field, a small magnetic field will be generated around the substance to interfere with the direction of original magnetic field, called chemical shift. The small magnetic field of paramagnetic substances and the original magnetic field follow the Curie's law, which imposes great influences on the measurement results of porosity. 


\section{Conclusion}

Based on the research of predecessors, the shale porosity determination methods are summarized and discussed and the following conclusions are made:

1). Kelvin principle is well used for the calculation of pore radius and pore diameter distribution in the nitrogen adsorption method and the selection of absorbent is of important significance to the accuracy of nitrogen adsorption method.

2). Constant-rate mercury intrusion method, as a emerging technology, has greater advantages and stronger adaption; the material hardness, sample preparation and contact angle impose great influences on mercury intrusion method.

3). Interval transit time and porosity have a linear relation and the relationship coefficient can be determined by experiment. In the acoustic wave test, the interval transit time is a decisive influencing factor.

4). In the nuclear magnetic resonance, CPMG method has a theoretical basis in the transverse relaxation determination of rock and can be used in the rock pore experiment. The main factors that affect the experimental results of nuclear magnetic resonance are instrument parameter, micro pore of sample and paramagnetic substance.

\section{References}

[1] Tian Hua, Zhang Shuichang, Liu Shaobo, Zhang Hong. Research of Pore Features of Rich Organic Shale by Mercury Intrusion Method and Gas Adsorption Method[J]. Acta Petrolei Sinica, 2012, 03:419-427.

[2] Tian Hua, Zhang Shuichang, Liu Shaobo, Ma Xingzhi, Zhang Hong. Optimization of Porosity Determination Parameter of Tight Reservoir[J]. Petroleum Geology \& Experiment, 2012, 03:334-339.

[3] Cui Jingwei, Zhou Caineng, Zhu Rukai, Bai Bin, Wu Songtao, Wang Tuo. New Progress of Shale Pore Research[J]. Advances in Earth Science , 2012, 12:1319-1325.

[4] Dou Feifei, Research on Features of Shale Reservoir at the Lower Strata of Longmaxi Group of East Sichuan[D]. China University of Mining \& Technology, 2014.

[5] Lu Juhong. Research on Gas Wetting Reversal Experiment and Numerical Simulation of Shale[D]. China University of Geosciences (Beijing), 2013.

[6] Li Yanan. Evaluation of Logging of Shale Gas Reservoir and Its Application[D]. China University of Mining \& Technology (Beijing), 2014.

[7] Ma Ruolong. Geologic Features of Mud Shale Strata and Shale Gas Exploitation Potential in Central Hunan, Southeast Hunan and Northeast Hunan[D]. Chengdu Univerisity of Technology, 2013.

[8] Guan Junfang, Hou Ruiyun. Features of Matrix Pore and Cleat Pore of Coal Reservoir and Porosity Determination Method[J]. Journal of North China Institute of Water Conservancy and Hydroelectric Power, 1999, 01:24-28. 